\title{
A LA BÚSQUEDA DE UN PINTOR BRUJENSE DEL RENACIMIENTO: ¿QUIÉN ES «MORAULUS»?
}

\author{
POR \\ DIDIER MARTENS \\ Université Libre de Bruxelles
}

\begin{abstract}
El retablo flamenco, fechado en 1561, de la iglesia de Santa María del Puerto de Santoña (Cantabria), lleva la inscripción Opus Petri Nicolai Morauli [...], acompañada de la dirección del pintor y de su lugar de residencia: Brujas. La misma firma se puede reconocer en la Misa de San Gregorio de Houston. En el presente artículo, el autor intenta demostrar que el pintor brujense que realizó estas pinturas no es otro que Pieter Claeissens el Viejo (hacia 1499/1576). Esta hipótesis, ya formulada por Weale en 1911, fue abandonada posteriormente frente a otra posible identificación (Pieter de Moor). Pero una datación estilística correcta de las obras firmadas por «Moraulus» y el examen de los archivos del Gremio de Pintores de Brujas hablan claramente a favor de la hipótesis de Weale.
\end{abstract}

Palabras clave: Pieter Claeissens el Viejo. Pieter de Moor. Moraulus. Santoña.

The Flemish altarpiece, dated 1561, from the church of Santa María del Puerto in Santoña (Cantabria), bears the inscription Opus Petri Nicolai Morauli [...], along with the artist's address and place of residence: Bruges. The same signature can be recognized on the Mass of Saint Gregory in Houston. In this study, the author attempts to demonstrate that the Bruges painter who realized these works is no other than Pieter Claeissens the Elder (c.1499/1576). This hypothesis, already formulated by Weale in 1911, was subsequently abandoned in favor of another possible identification (Pieter de Moor). Nevertheless, a correct stylistic dating of the paintings signed by «Moraulus» and examination of the archives of the Bruges Painter's Guild, clearly support Weale's hypothesis.

Key words: Pieter Claeissens the Elder. Pieter de Moor. Moraulus. Santoña.

Entre las numerosas controversias suscitadas en el estudio de la pintura flamenca del Renacimiento, la relativa a la identidad del misterioso pintor brujense 'Moraulus' merece una atención particular, por su antigüedad como por su carácter internacional. Ya hace más de un siglo que esta pregunta inquieta a los historiadores del arte alemanes, belgas y españoles, sin que ninguna de las soluciones propuestas hasta el momento haya logrado el consenso general.

Se han conservado por lo menos cinco obras con la inscripción Opus Petri Nicolai o Opus Petri Nicolai Morauli [...]. Dos de las cuales se conocen sólo por fotografía: el San Juan en Patmos (fig. 4), que pertenecía a la colección del barón de Brouwer en Ville-Pommeroeul (Bélgica, provincia del Hainaut) antes de ser vendido en 1947 en Bruselas ${ }^{1}$, y la Visitación, que en

${ }^{1}$ Óleo sobre tabla; 68 x $95 \mathrm{~cm}$. Vid., sobre esta obra, Schouteet, A., «Brugs schilder uit de 16de eeuw. Petrus Moraulus, geïdentificeerd», Studies over de kerkelijke en kunstgeschiedenis van West-Vlaanderen. Opgedragen aan Z.E.H. Michel English, Brujas, 1952, pp. 332-333.

AEA, LXXVII, 2004, 306, pp. 117 a 128 
1951 se encontraba en Londres, en la colección del conde Bobrinskoy ${ }^{2}$. Las otras tres tablas, por el contrario, son accesibles al historiador de arte. La Misa de San Gregorio fue adquirida en 1963 por la Fundación Sarah Campbell Blaffer de Houston ${ }^{3}$ (fig. 1) y recientemente presentada en Europa, en Francfort del Main, en una exposición interdisciplinar dedicada a la sangre $^{4}$. El monumental retablo de la iglesia de Santa María del Puerto de Santoña (Cantabria) es perfectamente visible en el brazo izquierdo del transepto (fig. 2). Fechado en $1561^{5}$, comprende seis paneles pintados, distribuidos en pares superpuestos. Los paneles que representan a San Jerónimo y San Sebastián llevan la firma del autor. Elisa Bermejo dió a conocer en 1974 este sorprendente retablo mural de tipo español, pintado por un artista flamenco ${ }^{6}$.

Todas las firmas conocidas de 'Moraulus' están precedidas por la palabra opus. El nombre del artista aparece pues en genitivo. Resulta una ambigüedad filológica: ¿el nominativo es Petrus Nicolaus Moraulus o Petrus Nicolai Moraulus? En el primer caso, el pintor sería Pedro-Nicolás como nombre propio, en el segundo caso, solamente Pedro y sería hijo de un tal Nicolás. Esta ambigüedad es, en parte, responsable de las dudas y vacilaciones de los investigadores.

La Visitación y el San Sebastián del retablo de Santoña llevan simplemente la inscripción Opus Petri Nicolai. En cambio, el San Jerónimo del mismo retablo, el San Juan en Patmos y la Misa de San Gregorio presentan una firma más larga: Opus Petri Nicolai Morauli Brugis in Flandria in platea quae dicitur den (H)ouden Sack, que puede traducirse como: Obra de Petrus Nicolai [o Nicolaus] Moraulus, residente en Brujas, en Flandes, en la calle llamada De Oude $\mathrm{Zak}^{7}$ (vid. fig. 3). Tenemos aquí un ejemplo único de la firma de un Primitivo flamenco que menciona, además del patronímico del pintor, su dirección, con el nombre de la villa, de la calle y del condado donde residía ${ }^{8}$. Podemos suponer que el artista, al actuar de esta forma, quiso asegurarse una cierta publicidad pues, tras leer la inscripción, el potencial cliente disponía de todas las informaciones necesarias para poder contactar con él.

El nombre de 'Moraulus' hace su entrada en la historia del arte en 1887. Este año, la Misa de San Gregorio Campbell Blaffer, procedente de la colección Von Aretin, se puso a la venta en Munich bajo la etiqueta Peter Nicolas Moraulus ${ }^{9}$. Para la ocasión, se publicó la fotografía de la tabla, que el vendedor califica de «bella e interesante», con transcripción del texto en latín y su traducción al alemán. La obra permaneció hasta 1947 como la única pintura conocida de 'Moraulus'.

\footnotetext{
${ }^{2}$ Óleo sobre tabla; 122 x $94 \mathrm{~cm}$. Foto RKD, La Haya, L22690.

${ }^{3} \mathrm{~N}^{\circ}$ Inv. 1963.1; óleo sobre tabla; $66,1 \times 77,8 \mathrm{~cm}$. Vid., sobre esta obra, Wright, C., A Golden Age of Painting. Dutch Flemish, German Paintings Sixteenth-Seventeenth Centuries from the Collection of the Sarah Campbell Blaffer Foundation, San Antonio, 1981, pp. 33-36; Clifton, J., The Body of Christ in the Art of Europe and New Spain, 1150-1800 (cat. exp.), Houston, Museum of Fine Arts, 1997-1998, n60.

${ }^{4}$ Bradburne, J.M. (ed.), Blut. Kunst, Macht, Politik, Pathologie (cat. exp.), Francfort del Main, Museum für Angewandte Kunst/ Schirn Kunsthalle, 2001/ 2002, pp.94, 201.

${ }^{5}$ Sólo han sido publicadas las dimensiones del panel que representa a Santiago: $106 \times 121 \mathrm{~cm}$. La fecha 1561 aparece dos veces en el remate de las calles laterales del retablo, en cifras romanas sobre el frontón izquierdo y árabes en el de la derecha.

${ }^{6}$ Bermejo Martínez, E., «Las tablas flamencas del retablo de la iglesia de Santa María, de Santoña», El arte de Flandes en Santander (cat.exp.), Santander, Instituto de Arte Juan de Herrera, 1974, s.p. y sobre todo eadem, «Un retablo flamenco en la iglesia de Santa María de Santoña», Archivo español de Arte, 49, 1976, n 193 , pp. 1-16.

${ }^{7}$ La calle De Oude Zak existe todavía en Brujas. Se halla en el barrio de la iglesia de Santiago. El nombre puede ser traducido como 'viejo culo de saco'.

${ }^{8}$ Entre los raros ejemplos de paralelos que podemos citar son destacables las tres firmas del pintor bruselense Colyn de Coter, que mencionan, además del nombre del artista, la villa de Bruselas, precisando que se encuentra en el ducado de Brabante. Vid., sobre este tema, Périer-D'Ieteren, C., Colyn de Coter et la technique picturale des peintres flamands du XVème siècle, Bruselas, 1985, p. 55.

${ }^{9}$ Katalog der nachgelassenen Gemälde-und Waffen-Sammlung des Freiherrn Carl von Aretin auf Haidenburg [...] (cat. subasta), Munich, Bayerische Vereinsbank, $1887, \mathrm{n}^{\circ} 100$.
}

AEA, LXXVII, 2004, 306, pp. 117 a 128 

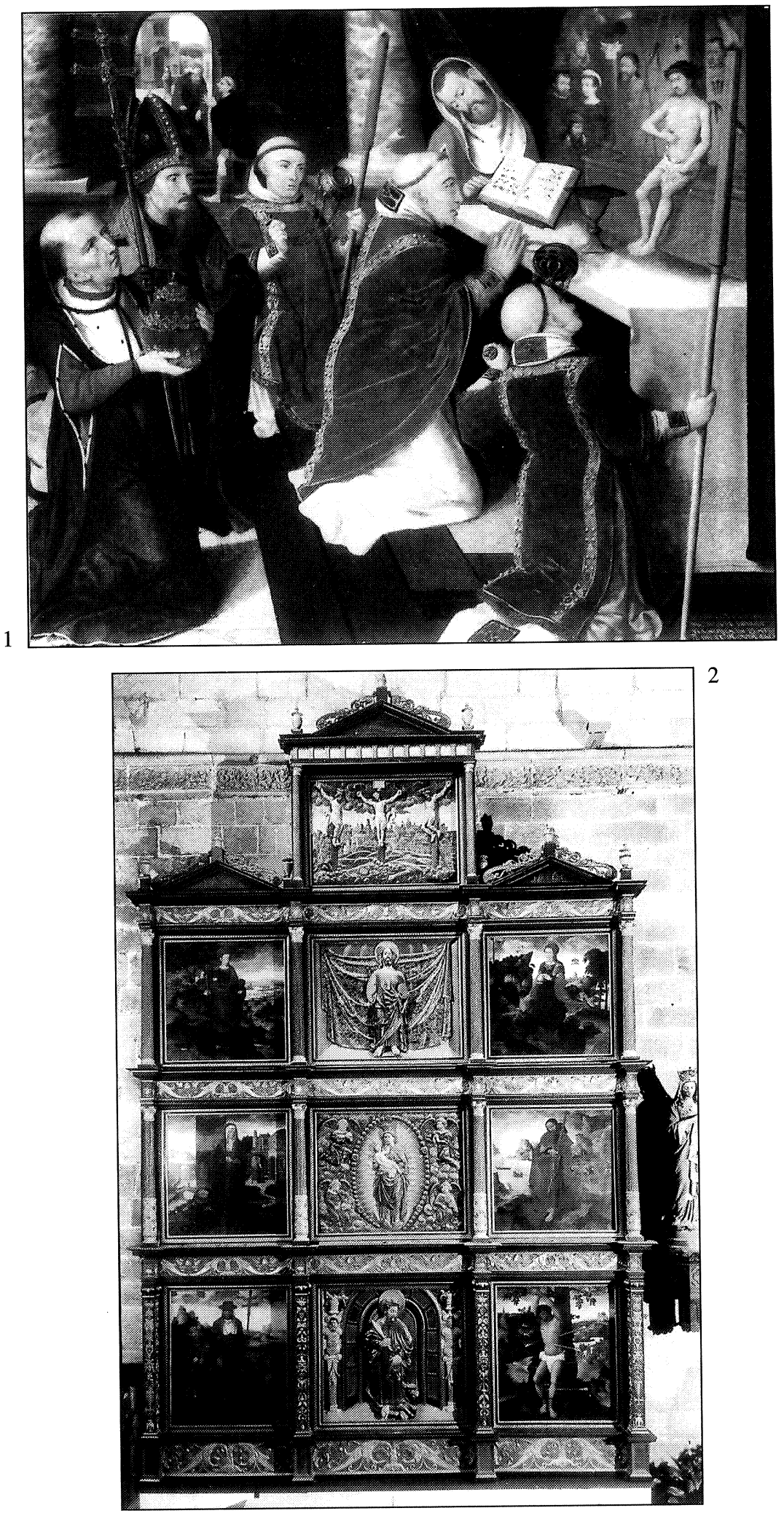

Fig. 1. 'Moraulus': Misa de San Gregorio; Houston, Fundación Sarah Campbell Blaffer (foto museo). Fig. 2. 'Moraulus': Retablo de San Bartolomé; Santoña, iglesia de Santa María del Puerto. 
Atestiguada por primera vez en el catálogo de venta Von Aretin, la idea de la existencia de un pintor brujense del Renacimiento que respondía al nombre de 'Moraulus' conoció una cierta fortuna entre los historiadores de arte. En 1888, Robert Stiassny sugirió su paternidad en el políptico flamenco conservado en el Museo Poldi Pezzoli de Milán ${ }^{10}$. Además, según este mismo

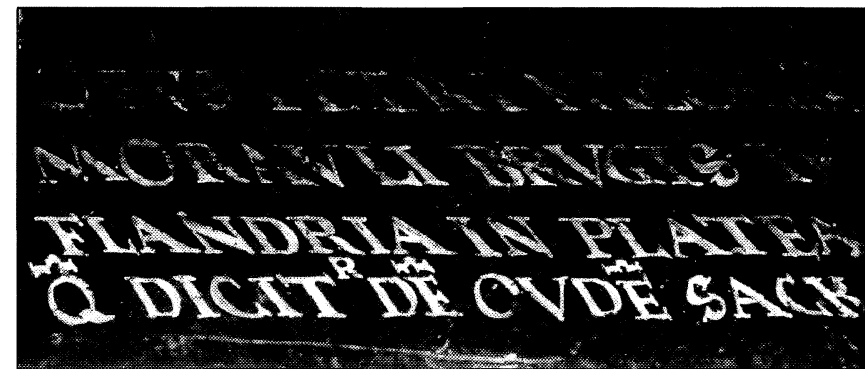

Fig. 3. 'Moraulus': Misa de San Gregorio (detalle); Houston, Fundación Sarah Campbell Blaffer (foto G.Berger, Bruselas). autor, sería ese brujense, «Moraul», el maestro del llamado Maestro del Retablo de San Bartolomé, uno de los principales pintores de Colonia de fines de la Edad Media. Las figuras del políptico de Milán presentarían estrechas semejanzas con el estilo del anónimo. Si bien ninguna de estas dos proposiciones ha sido mantenida por los historiadores del arte posteriores ${ }^{11}$, Alfred Von Wurzbach dedicó, en 1911, una breve entrada a 'Moraulus' en su diccionario de artistas flamencos y holandeses ${ }^{12}$. Ésta fue retomada en el Bénézit y se encuentra todavía en la edición de 1999. Pero, en esta última edición, 'Moraulus' aparece como pintor brujense del siglo XIX ${ }^{13}$...

¿El artista que firmó la Misa de San Gregorio Campbell Blaffer es verdaderamente una personalidad aparte? ¿Es el «homo novus en la historia del arte» del que hablaba Stiassny ${ }^{14}$ ? Algunos lo han dudado. Han transcrito el nombre del pintor bajo la forma Petrus Nicolai Moraulus - mejor que Petrus Nicolaus Moraulus, como lo había hecho el autor del catálogo de la subasta Von Aretin - y consideran Nicolai como la traducción latina del patronímico flamenco Claeissens. Esta interpretación, considerada ya desde 1910 por Von Wurzbach, a título de hipótesis, en su noticia sobre 'Moraulus', encontró un decidido abogado en los años siguientes, en la persona de James Weale ${ }^{15}$. Para Weale, que era entonces uno de los historiadores del arte más reputados en el campo de los Primitivos flamencos ${ }^{16}$, el artista que se escondía bajo el apelativo 'Moraulus' no sería en absoluto un homo novus. Por el contrario, se trataría de una personalidad bien conocida de los historiadores y archiveros brujenses, a saber Pieter Claeissens I (hacia 1499/1500-1576) ${ }^{17}$, el famoso Pieter el Viejo que fundó una verdadera di-

${ }^{10}$ Stiassny, R., «Altdeutsche und Altniederländer in oberitalienischen Sammlungen», Repertorium für Kunstwissenschaft, 11, 1888, pp. 391-393. Vid., sobre este políptico, Natale, M., en Museo Poldi Pezzoli. Dipinti, Milán, $1982, \mathrm{n}^{\circ} 219$.

${ }^{11}$ Vid., sobre el Maestro del Retablo de San Bartolomé, Budde, R./ Krischel, R. (ed.), Genie ohne Namen. Der Meister des Bartholomäus-Altars (cat. exp.), Colonia, 2001. El retablo del Museo Poldi Pezzoli fue atribuido por Friedländer al Maestro de la Leyenda de San Juan Evangelista, un anónimo brujense de hacia 1500. Vid. Friedländer, M.J., «Drei niederländische Maler in Genua», Zeitschrift für bildende Kunst, 61, 1927-1928, p. 278. Vid. también idem, Early Netherlandish Painting, VI: Hans Memling and Gerard David, Leyde/ Bruselas, 1971, 2, Add. 292.

${ }^{12}$ Von Wurzbach, A., Niederländisches Künstler-Lexikon [...], II, Viena/ Leipzig, 1910, p. 185.

${ }^{13}$ Bénézit, E., Dictionnaire critique et documentaire des peintres, sculpteurs, dessinateurs et graveurs [...]. Nouvelle édition entièrement refondue sous la direction de Jacques Busse, Paris, 1999, IX, p. 818.

${ }^{14}$ Stiassny, op.cit., p. 393.

${ }^{15}$ Vid. Weale, W.H.J., «Imaginary Flemish Painters», The Burlington Magazine, 20, p. 172; idem, «Peintres apocryphes Joseph de Bromere, Pierre Maroulus (sic)», Annales de la Société d'Émulation de Bruges, 62, 1912, pp. 62-63; idem, «Claeissins (Claeissens, Claeis, Claeiss) Pieter I», Allgemeines Lexikon der Bildenden Künstler von der Antike bis zur Gegenwart, VII, Leipzig, 1912, p. 33.

${ }^{16}$ Vid., sobre William Henry James Weale (1832-1917), Van Biervliet, L., Leven en werk van W.H.James Weale, een Engels kunsthistoricus in Vlaanderen in de 19de eeuw (=Verhandelingen van de Koninklijke Academie voor Wetenschappen, Letteren en Schone Kunsten van België. Klasse der Schone Kunsten, 55), Bruselas, 1991.

${ }^{17} \mathrm{He}$ adoptado la ortografía Claeissens, preferentemente, a la variante Claeissins de los autores flamencos más recientes (Dirk De Vos, Luc Devliegher, Eva Tahon). Vid, sobre la biografía de Pieter Claeissens el Viejo, Weale, W.H.J, «Peintres brugeois. Les Claeissins (1500-1656)», Annales de la Société d'Émulation de Brujas, 61, 1911, pp. 29-32; Tahon, E., «Pieter I Claeissens», Brugge en de Renaissance. Van Memling tot Pourbus. Catálogo (cat. exp.), Brujas, Memlingmuseum/ Oud-Sint-Janshospitaal, 1998, p.216.

AEA, LXXVII, 2004, 306, pp. 117 a 128 
nastía de pintores. Fue, en efecto, el padre de Pieter Claeissens II llamado Pierson (hacia 1530/ 1536-1612) y de Antoon (hacia 1536-1613), y abuelo de Pieter Claeissens III (muerto en 1623) y de Pieter Antuenis (muerto en 1608).

El patronímico flamenco Claeissens, que incluye un doble genitivo, significa 'de Nicolás' 18 y puede ser perfectamente traducido al latín por Nicolai. ¿Pieter Claeissens el Viejo habría entonces latinizado su nombre como Petrus Nicolai, siguiendo el ejemplo de tantos humanistas flamencos o alemanes del Renacimiento? El hecho que poseía, según los archivos, una tienda en la calle De Oude Zak y que vivía en 1550 en la calle Jan Mirael —de donde habría sacado el sobrenombre 'Moraulus' - avalaba, a los ojos de Weale, la identificación propuesta ${ }^{19}$.

A pesar del prestigio científico de Weale, esta identificación fue discutida, ya en 1952, por el archivero brujense Albert Schouteet ${ }^{20}$. Cinco años antes una segunda pintura de 'Moraulus' había salido a la luz: el San Juan en Patmos (fig. 4). Esta se puso a la venta en Bruselas, en 1947, bajo el nombre de «Claessens, Petrus I», lo que sugiere que el experto encargado de redactar el catálogo de la subasta conocía el artículo de Weale ${ }^{21}$. Apoyándose en esta pintura, que parece conocer personalmente, Schouteet afirma que su autor «debió vivir y trabajar a finales del siglo XV y comienzos del XVI» ${ }^{22}$ y concluye que «la obra del maestro Moraulus es sin duda alguna anterior al momento en que Pieter Claeissens llegó a ser pintor independiente», es decir en $1530^{23}$. Fue el parentesco, que no dejó de señalar, entre el San Juan en Patmos de la antigua colección de Brouwer y el pintado por Hans Memling, entre 1475 y 1479, sobre la puerta derecha del retablo de los Desposorios místicos de Santa Catalina ${ }^{24}$, lo que llevó al archivero a asignar una fecha tan antigua a la actividad de 'Moraulus'.

Si este último no es Pieter Claeissens el Viejo, ¿quién es? Tras examinar los años 1480 a 1521 en el registro de admisión A del Gremio de Pintores y Guarnicioneros de Brujas y señalar todos los Pieter que ejercieron la pintura, Albert Schouteet propone identificar a 'Moraulus' con un cierto Pieter de Moor. Debemos advertir que, contrariamente a Weale, Schouteet transcribe el nombre del artista bajo la forma Petrus Nicolaus Moraulus ${ }^{25}$. Pieter de Moor, que habría, según Schouteet, latinizado su apellido en 'Moraulus', sería nacido en Diksmuide (Dixmude), en Flandes, poco antes de 1469. Llegó a maestro en Brujas el 18 de febrero de 1500, donde murió en 1507, siendo enterrado en el cementerio de la iglesia de Santiago. Este detalle es importante porque, en este tiempo, la calle De Oude Zak se hallaba, en su mayor parte, dentro de la parroquia de Santiago.

La teoría de Albert Schouteet, que podía servirse de su autoridad como archivero de Brujas, suscitó la adhesión de numerosos especialistas. Fue aceptada, en 1963, por Simone Bergmans ${ }^{26}$, y después por Elisa Bermejo en sus dos primeros estudios sobre el retablo de Santoña, publicados en 1974 y $19766^{27}$, y seguida, igualmente, por Paloma Herrero en $1981{ }^{28}$ y por

\footnotetext{
${ }^{18}$ Herbillon, J./ Germain, J., Dictionnaire des noms de famille en Belgique romane et dans les régions limitrophes (Flandre, France du Nord, Luxembourg), Bruselas, 1996, p.173.

${ }^{19}$ La calle Jan Mirael existe todavía en Brujas. Se halla en el barrio de la iglesia de Santiago.

${ }^{20}$ Vid., sobre Albert Schouteet (1909-1991), Buysse, E., «Albert Schouteet. De mens en zijn werk», Album Albert Schouteet, Brujas, 1973, pp. 7-11; De Groote, J., «Bibliografie», Ibidem, pp. 13-22; Vandewalle, A., «In memoriam Albert Schouteet», Handelingen van het Genootschap voor Geschiedenis te Brugge, 128, 1991, pp. 129-134.

${ }^{21}$ Collection du baron de Brouwer. Catalogue de tableaux anciens des écoles flamande, hollandaise, française, anglaise et allemande du XVème au XVIIIème siècle [...] (cat. subasta), Bruselas, Palais des Beaux-Arts, 6/7 octubre 1947, $\mathrm{n}^{\circ} 46$.

${ }^{22}$ Schouteet, op. cit., p. 334.

${ }^{23}$ Schouteet, op. cit., p. 335.

${ }^{24}$ Schouteet, op. cit., p. 346.

${ }^{25}$ Schouteet, op. cit., pp. 340-343.

${ }^{26}$ Bergmans, S., en Le siècle de Bruegel. La peinture en Belgique au XVIème siècle (cat.exp.), Bruselas, Musées Royaux des Beaux-Arts, 1963, p. 78.

27 Vid. nota 6.

${ }^{28}$ Herrero, P., «El retablo flamenco de Santa María del Puerto de Santoña», Altamira, 43, 1981-1982, pp. 351-355.
} 
Enrique Campuzano, en las diversas noticias que ha ido dedicando al mismo retablo entre 1985 y $1999^{29}$. Una corta biografía de Pieter de Moor alias 'Moraulus' fue incluída en 1966 en un diccionario belga ${ }^{30}$

A pesar de su éxito, la hipótesis de Pieter de Moor tuvo también sus detractores. En 1989, el canónigo Steppe afirmaba, en una comunicación presentada en la sección flamenca de la Academia Real de Ciencias, Letras y Bellas Artes de Bélgica, que el retablo de Santoña había sido pintado por Pieter Claeissens el Viejo ${ }^{31}$, destacando: «Puesto que se trata de una obra datada precisamente, podría ser de una enorme utilidad para el estudio de [su] producción». El texto de esta comunicación, cuya edición ha sido anunciada desde 1989, no se publicó, de forma que desgraciadamente se ignoran los motivos que llevaron al canónigo Steppe a abandonar la teoría de Albert Schouteet.

En 1994, Luc Devliegher también rechazó la teoría del archivero brujense, pero ofrece una argumentación detallada ${ }^{32}$. Según él, Pieter de Moor no podría haber pintado el retablo de Santoña por dos razones. Por un lado, el panel que representa a San Sebastián lleva la inscripción Opus Petri Nicolai. Si el pintor hubiera tenido verdaderamente de Moor como apellido, se comprende mal como pudo omitir Moraulus en su firma. Además, el retablo está fechado en 1561 cuando, según los documentos, Pieter de Moor murió en 1507. El autor propone, como Steppe, volver a la hipótesis de Weale según la cual Petrus Nicolai - y no Nicolaus-Moraulus habría sido el patronímico latino de Pieter Claeissens el Viejo.

Esta nueva sugerencia fue bien recibida en España. Elisa Bermejo y Enrique Campuzano la han retomado en dos recientes noticias de catálogo ${ }^{33}$. Por el contrario, en Bélgica, los autores que últimamente se han interesado por Pieter Claeissens el Viejo dudan en atribuirle la paternidad de los cinco paneles firmados 'Moraulus' ${ }^{34}$. Lo cierto es que tras los años setenta, como resultado de los trabajos de Aquilin de Bisthoven y de Dirk de Vos, se ha admitido atribuir dos obras a Pieter Claeissens el Viejo: las puertas del abad Antoon Wydoot y el tríptico de Salamanca-Pardo ${ }^{35}$. Pero ambos conjuntos, conservados en el museo Groeninge de Brujas, no se parecen ni a los paneles de Santoña, ni a la Misa de San Gregorio Campbell Blaffer.

¿Qué podemos concluir de esta revisión de la literatura científica originada por el caso 'Moraulus'? Evidentemente que la identificación con Pieter de Moor debe ser definitivamente

${ }^{29}$ Campuzano Ruiz, E., El Gótico en Cantabria, Santander, 1985, pp. 530; idem, Arte de Flandes en Cantabria (cat exp.), Santillana del Mar, Torre de Don Borja, 1989, s.p.; idem, El retablo en Cantabria, Santander, 1999 , pp. 38-39.

${ }^{30}$ Schouteet, A., «Moor (Moraulus), Pieter (Petrus) de», Nationaal biografisch woordenboek, II, Bruselas, 1966, coll. 613-614.

${ }^{31}$ Steppe, J.K., «Een werk uit de Brugse nazomer: het altaarretabel van Santoña (Spanje, Cantabrische kust)», Jaarboek van de Koninklijke Academie voor Wetenschappen, Letteren en Schone Kunsten van België, 51, 1989 , p. 177.

${ }^{32}$ Devliegher, L., «De schilder Petrus Moraulus toch Pieter Claeissens I?», Handelingen van het Genootschap voor Geschiedenis, 131, 1994, ns 1-3, pp. 204-207.

${ }^{33}$ Bermejo Martínez, E., en Las tablas flamencas en la Ruta Jacobea (cat. exp.), Madrid, Fundación BSCH, 1999, n 65; Campuzano Ruiz, E., en: 2000 Anno Domini. La Iglesia en Cantabria (cat. exp.), Santillana del Mar, Museo Diocesano Regina Coeli, 2000, p. 122. En un artículo reciente («Een altaarstuk van de kartuize Sint-Anna-ter-Woestijne bij Brugge», Millenium, 14, 2000, pp. 128-134), Herman Ter Vrugt retoma igualmente la atribución del retablo de Santoña a Pieter Claeissens el Viejo (p. 130). Pero, proponiendo reconocer el patronímico de Moor en 'Moraulus', parece querer salvar también la teoría de Schouteet...

${ }^{34}$ Tahon, op.cit., p. 216. Vid. también Denhaene, G., «Claeissens Pieter I (Claeis, Claessens)», Le dictionnaire des peintres belges du XIVème siècle à nos jours [...], Bruselas, 1995, I, p. 177 y Tahon, E., «Claeissens (Claeis[s]; Claeis[s]ins), Pieter d.Ä.», Saur Allgemeines Künstler-Lexikon. Die bildenden Künstler aller Zeiten und Völker, XIX, Munich/ Leipzig, 1998 , p.346. Estas dos noticias del diccionario mencionan, en la bibliografía, el artículo de Elisa Bermejo de 1976, sobre el retablo de Santoña (vid. nota 6), pero no incluyen este conjunto en el catálogo de obras de Pieter Claeissens el Viejo.

${ }^{35}$ Vid., para las puertas del abad Wydoot, Janssens de Bisthoven, A., «De luiken van het altaarstuk met Antoon Wydoot, uit de Duinenabdij», Handelingen van het Genootschap voor Geschiedenis, 108, 1971, ${ }^{\text {os }} 3-4$, pp. 230-237; vid., para el tríptico de Salamanca-Pardo, De Vos, D., Stedelijke Musea Brugge. Catalogus schilderijen 15de en 16de eeuw, Brujas, 1979, pp. 99-100. La atribución de las dos obras a Pieter Claeissens el Viejo ha sido retomada por Tahon, Brugge en de Renaissance [...]. Catálogo, op.cit., p. 216.

$A E A$, LXXVII, 2004, 306, pp. 117 a 128 
abandonada, ya que se basa en una datación estilística errónea del San Juan en Patmos. Albert Schoutet vió una obra de la generación de Gerard David, Albrecht Cornelis y Jan Provoost ${ }^{36}$. El archivero y sus seguidores han subestimado evidentemente el fenómeno arcaizante en la pintura brujense del Renacimiento. Muchas obras, que se produjeron en Brujas hasta la época de Rubens, prolongaban, bajo formas apenas actualizadas, la tradición del siglo $\mathrm{xv}^{37}$. Resulta interesante, a este respecto, confrontar el San Juan en Patmos (fig.4) con una pintura del mismo tema que fue vendida en Bruselas en $1989^{38}$ (fig. 5). Firmada Petrus Claeiss(ens) me fecit, se trata probablemente de una obra de Pierson Claeissens ${ }^{39}$. La disposición de la composición, inscrita en un rectángulo horizontal, y la actitud del santo son muy parecidas. Los pies, el rostro y el brazo izquierdo, en particular, se disponen en ambas pinturas de manera análoga. Sólo el drapeado del manto y del vestido del evangelista, en el cuadro vendido en 1989, delata una fecha más avanzada. En lo demás, las dos obras parecen proceder de un mismo molde ${ }^{40}$. Ahora bien, la versión firmada Petrus Claeiss(ens) me fecit lleva la fecha de 1601.

¿Moraulus sería entonces Pieter Claeissens el Viejo? o ¿hay que volver a la hipótesis de Stiassny y considerar al autor de la Misa de San Gregorio Campbell Blaffer con una personalidad distinta dentro de la historia de la pintura brujense?

La identificación con Pieter Claeissens el Viejo tropieza con varias dificultades. Las objeciones 'estilísticas' que han sido recientemente formuladas no me parecen, sin embargo, decisivas. Ciertamente, es difícil reconocer la mano del autor de los paneles del retablo de Santoña en las puertas encargadas por el abad Wydoot y en el tríptico de Salamanca-Pardo. Pero no hay que olvidar que estas dos últimas obras no están firmadas y que su atribución a Pieter Claeissens el Viejo no se basa en ningún documento de archivo, sino únicamente en una serie de conjeturas, deducidas de la bibliografía de los comitentes. Según mi opinión, las puertas del abad Wydoot y el tríptico de los Salamanca-Pardo podrían ser perfectamente obras de Pieter Claeissens II. Por otra parte, ésta posibilidad ya fue propuesta para las puertas del abad Wydoot ${ }^{41}$.

Otras objeciones a la teoría de Weale parecen tener más peso. Luc Devliegher, que dudaba en identificar formalmente a Moraulus con Pieter Claeissens el Viejo, escribe: «es cuando menos sorprendente que Claeissens haya añadido a su nombre el de la calle donde él vivía» ${ }^{42}$. En efecto, según mis conocimientos, este caso sería único. Podemos preguntarnos si el curioso sobrenombre del artista deriva verdaderamente de Mirael. Desde el punto de vista de la morfología, el parentesco no es evidente ${ }^{43}$.

Más embarazoso para los partidarios de la teoría de Weale me parece el testimonio de la inscripción sobrepuesta a un retrato de la Galería Nacional de Oslo ${ }^{44}$ (fig. 6). El personaje re-

\footnotetext{
${ }^{36}$ Schouteet, op.cit., 1952, p. 346.

${ }^{37}$ Vid., sobre éste tema, Martens, D., «Transmission et métamorphose d'un modèle: la descendance au XVIème siècle de la 'Virgo inter virgines' attribuée à Hugo van der Goes», Annales de la Société Royale d'Archéologie de Bruxelles, 65, 2002, pp. 163-164, 171-172.

${ }^{38}$ Vid., sobre esta obra, Tableaux anciens et contemporains, mobilier, objets d'art, tapisseries, argenterie, bijoux (cat. subasta), Bruselas, Palais des Beaux-Arts, 7-9 noviembre 1989, $\mathrm{n}^{\circ} 641$.

${ }^{39}$ Eva Tahon ha llamado la atención recientemente sobre el hecho de que algunas obras firmadas Petrus Claeiss. posteriores a 1576 podrían ser de Pieter Claeissens II llamado Pierson, o de su hijo Pieter Claeissens III. Vid., sobre éstos dos pintores, Tahon, E., «Pieter II Claeissens, Pieter III Claeissens», Brugge en de Renaissance [...]. Catalogus, op.cit., pp. $224-225$.

${ }^{40}$ De la misma matriz procede también un San Juan en Patmos que figura en la puerta derecha de un tríptico de Brujas conservado en una colección privada española. Vid., sobre esta obra, Bermejo Martínez, en Las tablas flamencas, op.cit., $\mathrm{n}^{\circ} 66$.

${ }^{41}$ Janssens de Bisthoven, op. cit., p. 237.

${ }^{42}$ Devliegher, op. cit., p. 206, nota 12.

${ }^{43}$ Además de la forma Mirael, Albert Schouteet (De straatnamen van Brugge. Oorsprong en betekenis, Brujas, 1977, p. 92) menciona también las formas Merael, Marael, Simmerol, Jemerol, Amarael, Ammirael y Admirael. Ninguna se asemeja a 'Moraulus'.

${ }^{44}$ Vid., sobre esta obra, Nasjonalgalleriet. Katalog over utenlandsk malerkunst, Oslo, 1973, $\mathrm{n}^{\circ} 384$; Tahon, E., en Brugge en de Renaissance. Van Memling tot Pourbus. Notities (cat. exp.), Brujas, Memlingmuseum/ Oud-Sint-Janshospitaal, $1998, \mathrm{n}^{\circ} 118$.
} 

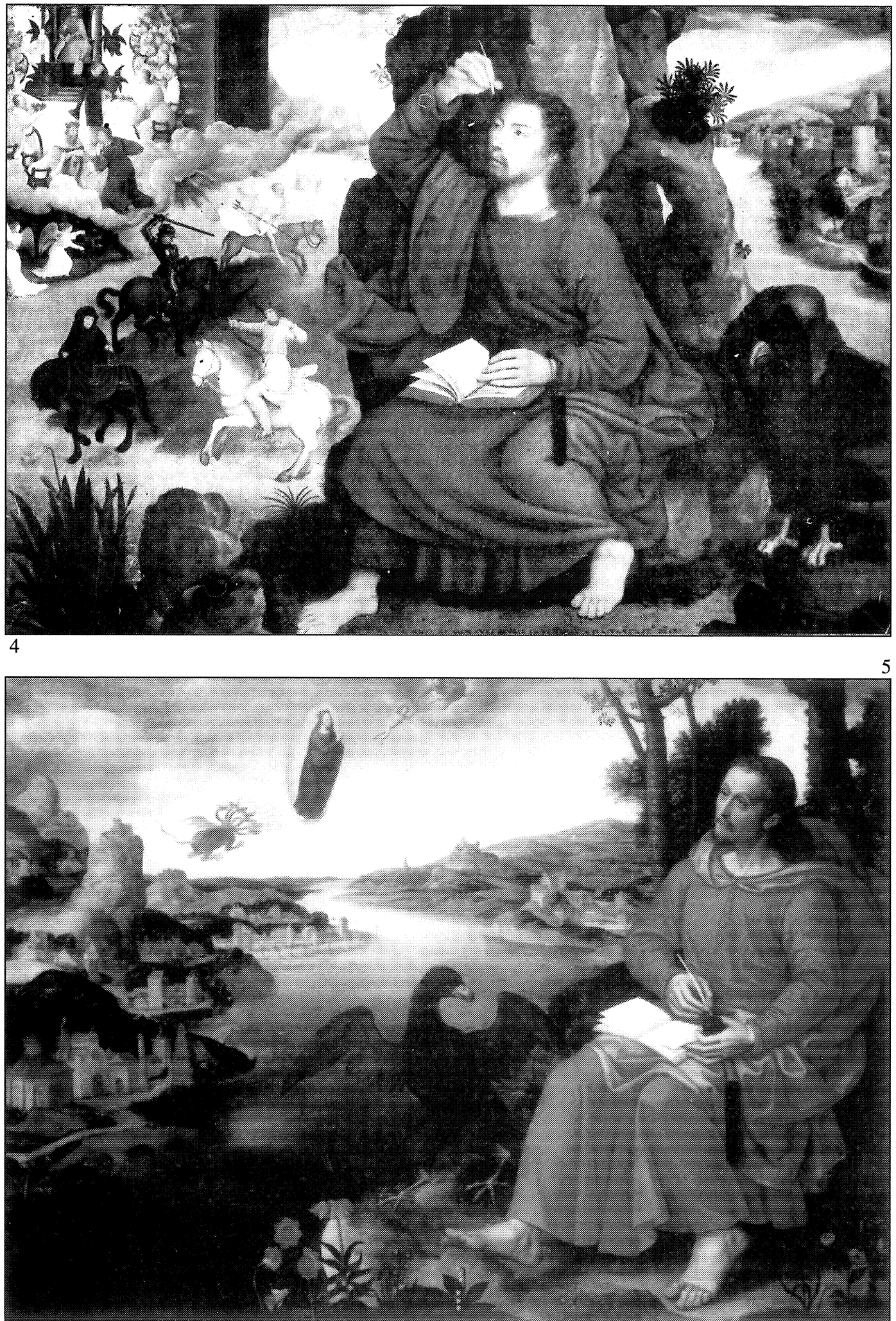

Fig. 4. 'Moraulus': San Juan en Patmos; Bruselas, subasta, 6 octubre 1947 (foto RKD, La Haya).

Fig. 5. Pieter Claeissens II (?): San Juan en Patmos; Bruselas, subasta, 7-9 noviembre 1989.

AEA, LXXVII, 2004, 306, pp. 117 a 128 
presentado - un hombre maduro - está rodeado de un pseudo-encuadramiento de estilo renacentista que lleva la inscripción Petrus Claeiss(ens) Pic(tor). Encima de su cabeza puede leerse la fecha de 1560 y su edad: 60 años. Los historiadores del arte han reconocido, desde hace ya mucho tiempo, una efigie de Pieter Claeissens el Viejo en este cuadro. ¿Se trata de un autorretrato? No podemos afirmarlo con certeza, como lo destacan Dirk de Vos y Eva Tahon ${ }^{45}$, pero, parece claro que estamos en presencia de una imagen que reproduce los rasgos del artista. No hay, en efecto, ninguna razón para dudar de la autenticidad de la inscripción, insertada en el simulacro de moldura dorada, pintada directamente sobre la tabla. Este tipo de marcos era muy apreciado en Brujas en el siglo XvI.

Entonces ¿qué constatamos? Que la inscripción identificando al artista está hecha en latín y que el mismo nombre ha sido latinizado, pero el apellido continua siendo Claeiss(ens). ¿Por qué no emplear la forma Nicolai? Además, si Pieter Claeissens el Viejo y 'Moraulus' son uno mismo, podría haberse atenido a que este seudónimo del artista figurara también en el retrato de Oslo. ¿Éste no representa a Pieter Claeissens el Viejo «en tanto que pintor», como dice la inscripción?

Observamos que Pierson y Antoon Claeissens firmaron en repetidas ocasiones sus obras en latín, sin utilizar jamás el patronímico Nicolai. ¿Si realmente su padre hubiera desarrollado su carrera bajo este nombre, no es sorprendente que ellos no lo hayan adoptado a su vez?

Sin embargo, estamos convencidos del hecho de que es Pieter Claeissens el Viejo, y ninguno otro, el que firmaba Petrus Nicolai Moraulus. Resulta decisivo un argumento que nos aporta el examen combinado de dos fuentes que emanan del Gremio de Pintores y Guarnicioneros de Brujas.

En primer lugar, se trata del registro de admisión A, en el que se apoyó Albert Schouteet para identificar a 'Moraulus' con Pieter de Moor, pero él se limitó a consultar los años 1480$1521{ }^{46}$. Brujas es una de las raras ciudades europeas que han conservado, para los siglos XV y XVI, una lista casi completa, año por año, de los pintores que accedieron al grado de maestro ${ }^{47}$. Esta lista -el registro A- cubre el período 1453-1578. Hay que lamentar unicamente las interrupciones entre 1493-1494 y 1498-1499 en las que las nuevas inscripciones de los maestros no figuran ${ }^{48}$.

'Moraulus', para poder ejercer su profesión de pintor en Brujas, tuvo seguramente que inscribirse en la Corporación local de Pintores y Guarnicioneros. No vemos razón alguna por la cual él pudiera haber sido dispensado de este trámite. A juzgar por las inscripciones detalladas que el pintor ponía sobre sus obras, nada nos indica que fuera noble, eclesiástico o 'pintor del rey'. Hay que esperar, por lo tanto, encontrar a 'Moraulus' en este registro A. El año de 1561 ha sido tomado como terminus ante quem para la búsqueda. El artista debía ser ya primer maestro pues ejecutó el retablo de Santoña, y podemos suponer que en este momento debía tener, como máximo, ochenta años. Debió obtener el grado de maestro, como muy pronto, en 1500 .

El registro A ha sido examinado para el período 1500-1561 y todos los pintores con el nombre de Pieter, o derivados, se han señalado. Encontramos a: Pieter Christus (maestro en 1500-1501; es Pieter Christus II, el hijo menor del célebre Petrus Christus); Pieter Casboot (1508); Pieter van de Wyvere (1509); Pieter Reylof (1518); Pierson Puseel (1518); Pierkin van Necke (1520); Pierkin de Clyever (1525); Pieter Claeys (1529, es Pieter Claeissens el Viejo,

${ }^{45}$ De Vos, op.cit., p. 102; Tahon, en: Brugge en de Renaissance [...]. Notities, op.cit., $\mathrm{n}^{\circ} 118$.

46 SAB, Oud Archief, reeks 314, Beeldenmakers, Register A.

${ }^{47}$ Vid., para una edición de esta lista, Vanden Haute, C., La corporation des peintres de Bruges, Brujas/ Courtrai, 1913.

${ }^{48}$ Vid., sobre este tema, Schouteet, A., De Vlaamse Primitieven te Brugge. Bronnen voor de schilderkunst te Brugge tot de dood van Gerard David. Vol.1: A-K (=Fontes Historiae Artis neerlandicae, 2), Bruselas, 1989, p. 8. 


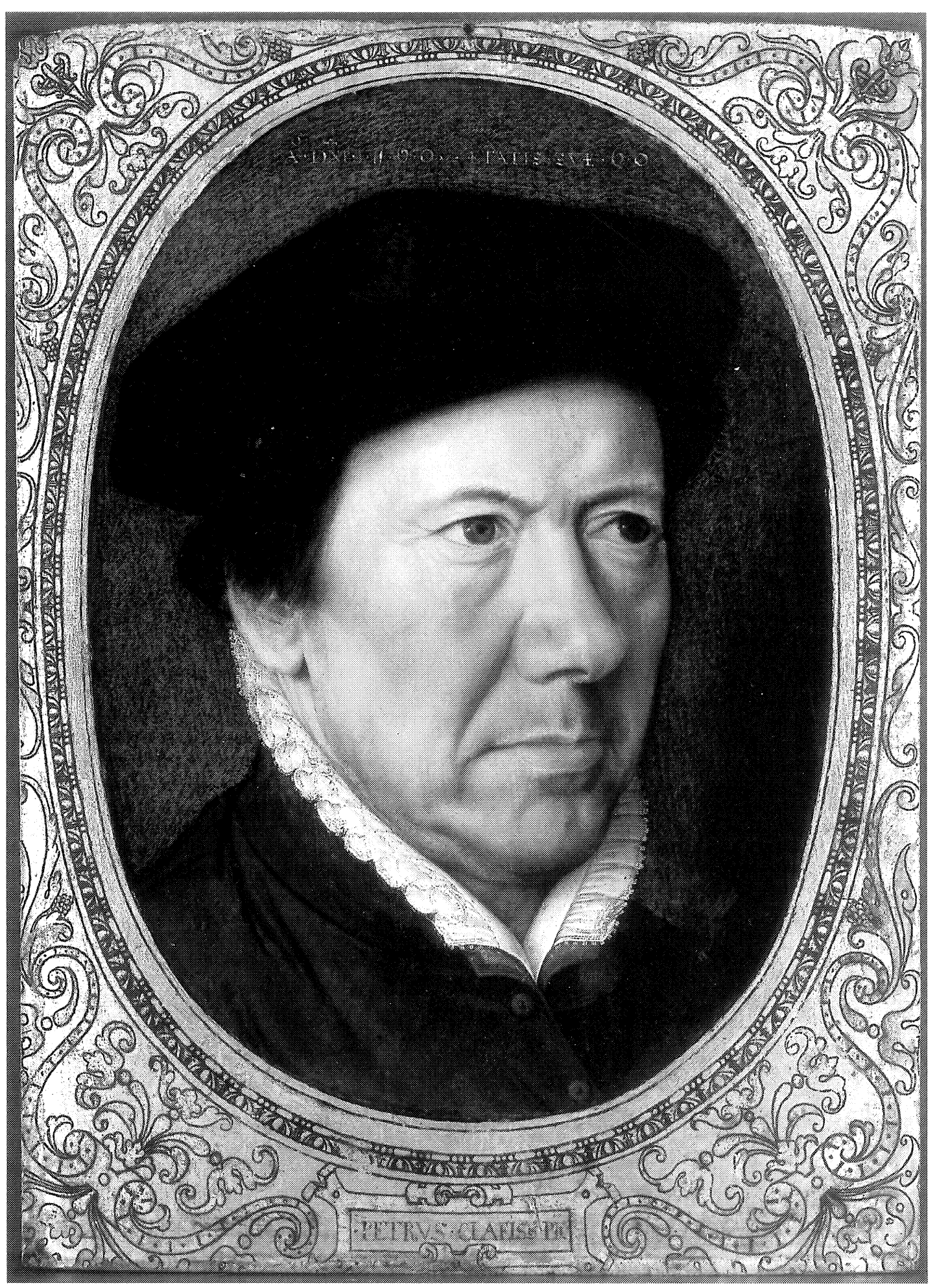

Fig. 6. Pieter Claeissens I (?): Retrato de Pieter Claeissens I; Oslo, Nasjonalgalleriet (foto museo). 
como indica una nota manuscrita ${ }^{49}$ ); Pieter Jansyns Poerbus (1543, es el famoso Pieter Pourbus); Pietere vanden Boeyerie (1548) y Pietere Malynnes (1550). Como se puede constatar, si exceptuamos a Pieter Claeys, ninguno de los Pieter encontrados lleva un apellido cuya forma latinizada podría ser Nicolaus, Nicolai o Moraulus. ¿Hay que concluir entonces que Pieter Claeissens el Viejo es el misterioso 'Moraulus'?

Teóricamente, una duda aún permanece. Albert Schouteet ha llamado la atención sobre el hecho de que algunos raros pintores brujenses, conocidos por los documentos de los siglos $\mathrm{XV}$ y XVI, no figuran en el registro $\mathrm{A}^{50}$. Los escribanos de la corporación habrían sido, a veces, negligentes. ¿La identificación de Pieter Claeissens el Viejo con 'Moraulus' debe seguir siendo sólo una hipótesis?

Es posible rellenar las probables lagunas del registro A gracias a una segunda fuente, el obituario de la Corporación de Pintores y Guarnicioneros de Brujas ${ }^{51}$. Contiene la lista de los maestros que estaban inscritos en el gremio en el momento de su muerte ${ }^{52}$. Esta lista -una especie de memorial - abarca el período de 1450-1801, sin ninguna interrupción. Los pintores se hallan clasificados por orden cronológico, según la fecha de su defunción. Suponemos que algunos maestros que figuran en el registro A, pero no en el obituario, debieron abandonar Brujas antes de su muerte ${ }^{53}$.

Tomando como base este obituario, hemos efectuado un segundo test para identificar 'Moraulus' en los archivos de la Corporación de Pintores y Guarnicioneros. Esta vez, la fecha de 1561 ha sido tomada como término a quo de la búsqueda. Se ha considerado que si 'Moraulus' tenía veinte años en 1561, cuando terminó el retablo de Santoña, su muerte debió sobrevenir, como muy tarde, hacia 1640 . El obituario ha sido examinado para el período de 1560 1640. Todos los pintores que tienen como nombre Pieter y sus derivados se han anotado. Asi aparecen: Pieter Puseel, Pieter Malynis, Pieter Claeissins (es Pieter Claeissens el Viejo; estos tres nombres ya se hallaban en el registro A para el período 1500-1561); Pieter Pourbusse (es el famoso Pieter Pourbus), Pieter Anthuenis Claeissens (es el hijo de Antoon Claeissens), Pierson Claeyssins (es Pieter Claeissens II, llamado Pierson); Pieter Dutoy, Pieter Claeissins (es Pieter Claeissens III, hijo de Pieter Claeissens II) y Pieter van Hove. Como se constata, si exceptuamos a los miembros de la dinastía Claeissens, ninguno de los Pieter encontrados lleva un apellido cuya forma latinizada pueda ser Nicolaus, Nicolai o Moraulus.

'Moraulus' no puede, evidentemente, ser Pieter Claeissens II, del que conocemos bien su estilo, más evolucionado que el de los paneles del retablo de Santoña. No puede ser, a fortiori, Pieter Claeissens III, que era el hijo de Pierson y prolongó la manera de hacer de su padre. 'Moraulus' no puede ser tampoco Pieter Antuenis Claeissens, muerto en 1608. Las raras obras firmadas por este pintor se caracterizan por un estilo próximo al de su padre Antoon, una manera indiscutiblemente más moderna que la de las obras firmadas 'Moraulus' ${ }^{54}$. Queda el «Maestro Pieter Claeissens, pintor e iluminador». Sin ninguna duda, se trata de él, el misterioso Petrus Nicolai.

Esta conclusión sorprenderá, a la vista del testimonio facilitado por la inscripción del retrato de Oslo. Es posible que el artista no hubiera firmado siempre Petrus Nicolai. ¿Habría

\footnotetext{
${ }^{49}$ «Pieter Claeissins, den alder ouchten; naer ghecomen is Gillis, Pieter ende Anthunis (Pieter Claeissens, el más anciano; descienden de el Gillis, Pieter el Joven y Antoon)».

${ }^{50}$ Schouteet, op.cit., 1989, pp. 7-8.

${ }^{51} \mathrm{SAB}$, Oud Archief, reeks 314, Beeldenmakers, Obituarium.

${ }^{52}$ Vid., sobre esta lista, Geirnaert, N., Dood maar niet vergeten: de gerestaureerde memorielijst van de Brugse schilders, Brujas, 2001. Vid., para una edición de la lista, Vanden Haute, op.cit., pp. 194-210. La lista se encuentra también en www.brugge.be/archief.

${ }^{53}$ Schouteet, op. cit., 1989, pp. 6-7.

54 Vid., por ejemplo, los Excesos de la Humanidad antes del Diluvio (De Mirimonde, A.P., «La musique chez les peintres de la fin de l'école de Bruges», Jaarboek van het Koninklijk Museum voor Schone Kunsten, 1976, pp. 77-78).
} 
reservado la forma latina de su apellido para las obras realizadas para clientes españoles, considerando que esta forma sería más fácil de memorizar, para un castellano, que Claeissens? Ignoramos el origen de la Misa de San Gregorio y del San Juan en Patmos. Por el contrario, el retablo de Santoña ha permanecido in situ, en la iglesia para la cual fue concebido. El uso diferenciado del nombre Nicolai permitiría explicar porque éste no aparece en la inscripción del retrato de Oslo, una obra de carácter privado destinada a la descendencia del artista y no al mercado extranjero.

¿Habrían tenido los hijos de Pieter el Viejo menos ambiciones internacionales que su padre? Abandonaron el nombre Nicolai y, en las ocasiones en que trabajaron para clientes españoles, firmaron sólo con su patronímico flamenco ${ }^{55}$. Debemos puntualizar que ellos tuvieron menos razones que su padre de preocuparse del mercado extranjero. La actividad de Pieter Claeissens el Viejo coincide con la gran crisis iconoclasta que obligó a los artistas del norte de Europa a buscar nuevas salidas para sus producciones, mientras que Pierson y Antoon Claeissens conocieron, ambos, el siglo xVII. A partir de 1585, tuvieron el privilegio de trabajar en el contexto de la Pax hispanica, ciertamente más favorable a los imagineros flamencos que el de la época de los Gueux. Estimulados por el restablecimiento del catolicismo, como única religión tolerada y por la necesidad de reemplazar las obras destruidas o vendidas bajo el régimen calvinista, la demanda de pinturas religiosas conoce, desde finales del siglo xvI, en Flandes y en Brabante, una sensible recuperación ${ }^{56}$, de la cual Pierson y Antoon Claeissens evidentemente se beneficiaron.

Podemos decir que el destino habría jugado una mala pasada a Pieter Claeissens el Viejo. $\mathrm{El}$ artista se esforzó para dar a su actividad como pintor una 'visibilidad' particular, utilizando de forma persistente el latín, la lingua franca de las élites del siglo xvi. Esta 'estrategia', que le llevó a latinizar su patronímico flamenco, para constituir una especie de label identificable por el público internacional, se volvió en su contra. Mientras que desde la segunda mitad del siglo XIX, las producciones firmadas por Pierson y Antoon Claeissens fueron restituidas a sus respectivos autores, previamente identificados en los archivos brujenses ${ }^{57}$, las pinturas firmadas Petrus Nicolai, por el contrario, han permanecido huérfanas demasiado tiempo. Pese a la intuición correcta de Weale, no han servido de base, hasta ahora, para la reconstitución de la obra de Pieter Claeissens el Viejo.

\section{Agradecimientos}

Es un agradable deber el dar las gracias a aquellas personas que me han ayudado en la preparación de este artículo: el Reverendo Padre Francisco Hoyo Ceballo (Santoña), James Bradburne (Francfort del Main), Enrique Campuzano Ruiz (Santillana del Mar), James Clifton (Houston), Anna Maria Coderch (Fribourg), Marta Negro Cobo (Burgos), que ha traducido mi texto francés, y André Vandewalle (Brujas). Durante mis investigaciones he disfrutado, como de costumbre, de la hospitalidad del Centre d'étude de la Peinture du XVème siècle dans les Pays-Bas méridionaux et la Principauté de Liège (Bruselas).

\footnotetext{
${ }^{55}$ Así sucede en las figuras de San Juan Bautista y de un Santo obispo que se hallaban antiguamente en la Cartuja de Burgos y que estaban firmadas Antonius Claesins. Vid., sobre el tema, Ponz, A., Viage de España, XII, 11 (= éd. Aguilar, Madrid, 1988, 3, p. 574). Vid. también un San Francisco recibiendo los estigmas firmado por Pieter Claeissens II (o III?), fechado en 1602 en la iglesia de los franciscanos de Zarauz (Guipúzcoa). La obra está reproducida por Fernández Pardo, F., en Las tablas flamencas, op.cit., p. 174.

${ }_{56}$ Vid., sobre el tema, Vlieghe, H., Flemish Art and Architecture 1585-1700, New Haven/ Londres, 1998, p. 4.

57 Vid., por ejemplo, Siret, A., «Claes, Claessens, Claeis ou Claeissens (Antoine)» y «Claes, Claessens, Claeis ou Claeissens (Pierre)», Biographie nationale, IV, Bruselas, 1873, coll. 124-128.
}

$A E A$, LXXVII, 2004, 306, pp. 117 a 128 